\title{
Tensiones y paradojas en la configuración de la identidad ciudadana en la escuela secundaria*
}

\author{
Lucía Elena Rodríguez Mc Keon**
}

\section{Resumen}

En este trabajo se muestran algunos de los hallazgos más significativos de una investigación realizada en una escuela secundaria con el propósito de analizar los procesos de identificación ciudadana que se organizan en la escuela. A partir de la recuperación de datos cualitativos a través del estudio en caso, se analizan algunas de las problemáticas que atraviesa la configuración de la subjetividad ciudadana en los jóvenes a través de la formación escolar.

\section{Palabras clave}

Escuela secundaria - Procesos de identificación ciudadana Formación.

\section{Abstract}

In this paper some of the most significant findings of an investigation conducted at a high school in order to analyze the processes of citizen identification that promotes school in this time of change is. From qualitative data recovery through the case study, discusses some of the problems running through

* Artículo recibido el 21 de Mayo de 2015. Aceptado el 2 de Julio de 2015.

** Lucía Elena Rodríguez Mc Keon es Doctora en Educación por la Universidad Autónoma del Estado de México. Es investigadora de la Universidad Pedagógica Nacional.

Correo electrónico: luciamckeon@gmail.com 
the configuration of citizen subjectivity in young people through school education.

\section{Keywords}

High School - citizen identification processes - Formation.

\section{Introducción}

Este artículo forma parte de una investigación más amplia orientada a analizar los procesos de identificación ciudadana que en la actualidad se propician en la escuela secundaria. El interés de investigar acerca del tema se relaciona con la necesidad de contar con estudios diagnósticos de lo que sucede actualmente en la escuela secundaria como espacio localizado de formación de ciudadanía, frente a las profundas transformaciones que se producen en nuestra sociedad a partir de la experiencia de la globalización, que, en el caso de nuestro país, se han entretejido con el debilitamiento de algunos de los referentes que por años dieron sustento a la formación de ciudadanía en la escuela.

Para ello, nos propusimos reconstruir la trama significativa que se revela en el discurso de los sujetos en torno a las prácticas de formación de ciudadanía que se propician en la escuela, a fin de reconocer el tipo de identificaciones ciudadanas que contribuyen a configurar

Al concebir que la formación de ciudadanía implica la construcción de procesos de identificación ciudadana, en esta investigación se examinaron algunos de los significados que producen, circulan y dan sentido a la arquitectura del dispositivo escolar, en el entendido de que allí se condensan maneras específicas de dinamizar la construcción del nosotros, de procesar la diferencia y de delimitar el margen de intervención de los sujetos en la producción y modificación del orden social, lo que da como resultado la configuración de determinados modos de reconocerse como ciudadanos.

Este posicionamiento relativo al reconocimiento de la existencia de una relación indisoluble entre los procesos de formación y la construcción de identidad ${ }^{1}$ nos obligó a asumir una perspectiva de análisis que se planteó que trascendiera los aspectos de orden didáctico y curricular involucrados en las definiciones declarativas contenidas en los planes y programas de estudio con relación a las temáticas de la formación para la ciudadanía, situándonos en un plano relacionado con la producción de la racionalidad

\footnotetext{
${ }^{1}$ En el horizonte reflexivo que sostiene nuestra argumentación, las nociones de formación e identificación ciudadana se encuentran profundamente imbricadas. Lo anterior debido a que, tal como se desarrolla en el texto, los procesos de formación en el campo de la ciudadanía no solo involucran la adquisición de saberes, sino que se caracterizan porque suponen modos de reconocimiento de sí como ciudadano que son legitimados políticamente al interior de una comunidad determinada.
} 
pedagógica que da sentido a los discursos y prácticas que habitan al interior de la escuela como espacio formativo.

La vía para recoger y trabajar los datos fue de tipo cualitativo y de corte inductivo. El referente empírico lo constituye una escuela secundaria general de turno matutino ubicada en el sur de la Ciudad de México que, por los resultados académicos que obtiene y su normativa, es considerada como una buena escuela. El método de análisis se basó en lo que Popkewitz (1998: 11) llama "etnografía crítica". Esta se centra en la búsqueda de las reglas y normas a partir de las cuales se construye el significado. El trabajo de acopio de datos se basó en la observación de prácticas e interacciones en el lugar y en la realización de cuatro entrevistas en profundidad a maestros, además de la realizada a la directora y a diez alumnos que cursan el tercer grado

Para la selección de los docentes se utilizó el criterio de casos extremos (Goetz y Lecompte 1988): se buscó a quienes recibían los calificativos de "buen maestro" y "mal maestro" en razón de su apertura y disposición para acompañar el proceso formativo de los jóvenes; mientras que en el caso de los alumnos, el número se limitó a partir del criterio de exhaustividad.

\section{La mirada: las identificaciones ciudadanas y el entramado del saber-poder}

Desde nuestro punto de vista, los procesos de identificación ciudadana en la escuela secundaria se despliegan en el eje tensional que se produce entre unidad y diferenciación en las prácticas formativas que se propician en el dispositivo escolar. Esta tensión favorece o impide la posibilidad de dar cabida a la subjetividad y la diferencia como rasgos distintivos de un modo de ser ciudadano y de asumir el ejercicio que dicho reconocimiento implica.

Con base en Dubar consideramos que los procesos de construcción de identificaciones ciudadanas se configuran al interior de dinámicas relacionales que implican siempre la alteridad y que, por lo tanto, resultan contingentes al estar sujetas a variación histórica. Al rechazar que existan pertenencias 'esenciales', el autor reconoce que lo que existe son modos de identificación, ${ }^{2}$ variables en el curso de la historia colectiva y de la vida personal; afiliaciones a diversas categorías que dependen del contexto, por lo que desde esta perspectiva no hay identidad sin alteridad: "las identidades, tanto como las alteridades, varían históricamente y dependen del contexto de su definición" (2002: 12).

Tomamos en cuenta que todo proceso identificatorio recurre a palabras, categorías y referencias socialmente identificables y toda forma identitaria es inseparable de una forma de poder, de relación social y de alteridad (Dubar, 2002). Por ello, tiene una

\footnotetext{
${ }^{2}$ Recordemos que Dubar distingue entre identificaciones reivindicadas o "para sí" y atribuidas o "para otro".
} 
dimensión política; solo puede desarrollarse si la legitimidad del poder está objetivamente garantizada y es subjetivamente significante.

Por otro lado, con el sustento de la perspectiva de Foucault (2003), consideramos que los procesos de identificación ciudadana que se propician en la escuela se realizan en un campo de experiencia a partir del cual los individuos se reconocen como sujetos ciudadanos. La experiencia se construye articulada con un sistema de reglas y restricciones que se produce al interior de una cultura, en la correlación entre campos de saber, tipos de normatividad y formas de subjetividad que, como efectos de poder, delimitan en un momento determinado los márgenes de normalidad existentes en torno al ejercicio ciudadano y a lo posible de ser pensado en el campo, en tanto acción y participación del sujeto en un orden social y político. Para este autor, la construcción de identidades se encuentra profundamente imbricada al interior de los juegos de saber-poder. Este proceso se realiza mediante 'prácticas divisorias' que objetivizan a los sujetos, situándolos en espacios calculables que designan su propia individualidad, imponiéndoles una ley de verdad que deben reconocer y que los otros deben reconocer en ellos. Si se entiende la producción de subjetividad en ese espacio, se visualiza no solo el sometimiento al otro en términos de control y dependencia, sino también se ven las maneras en que el sujeto se reconoce a sí mismo, a partir de un régimen de verdad que lo deja "atado a su propia identidad" (Foucault, 1988: 231).

Las prácticas divisorias se organizan mediante procedimientos que Foucault llama "estrategias". 3 Estas son la expresión de las tecnologías de gobierno de la individualización, se construyen como resultado de juegos de acción y reacción, de pregunta y respuesta, de dominación y retracción y también de lucha (Foucault, 1998) y se encuentran siempre sujetas a la modificación que se produce como resultado de dichos juegos.

En este contexto, lo que denominamos 'estrategias de identificación ciudadana' son las formas en que se organizan las relaciones de saber-poder al interior del dispositivo escolar de formación de ciudadanía para favorecer que los jóvenes se asuman como ciudadanos delimitando márgenes de acción determinados sobre sí mismos, con los otros y el mundo, a partir de juegos de alteridad que se despliegan a través de mecanismos de gestión cultural y política de la diferencia. Como tales, las estrategias se sostienen a partir de determinados sistemas exclusionarios que responden a las finalidades que orientan al dispositivo, las cuales pueden variar históricamente dependiendo de la manera en que se construyen los márgenes que autorizan lo posible

\footnotetext{
3 Usamos el término "estrategia" recuperando dos de las acepciones que le otorga Foucault (1988: 242-243). Por un lado, el sentido que designa la elección de los medios empleados para obtener un fin, es decir, la racionalidad utilizada para alcanzar los objetivos, y por el otro, el conjunto de procedimientos para privar al enemigo de sus medios de combate, obligándolo a renunciar a la lucha y obtener así la victoria.
} 
de ser pensado. En el siguiente apartado damos cuenta de las finalidades que orientan dicho proceso y del tipo de estrategias que pudimos reconstruir a partir del análisis.

\section{"Ser alguien en la vida"}

A partir del análisis realizado, fue posible identificar la paradoja que atraviesa a la escuela como dispositivo de construcción de identificaciones ciudadanas. Mientras nuestras sociedades transitan por un momento en el cual los procesos de desocialización dificultan la pervivencia de matrices de identificación ciudadana inscritas en grandes totalidades, encontramos que la racionalidad dominante en el dispositivo de formación de ciudadanía en la escuela se empeña en sostener que los jóvenes logren "ser alguien en la vida", en el marco de esas matrices.

Así lo vimos cuando en un primer momento del análisis identificamos la manera como las maestras y maestros significan las intencionalidades de la formación de ciudadanía:

"Formar a los jóvenes como ciudadanos es que aprendan a respetar a la patria, nuestras instituciones y sus leyes, pero también que participen en el mejoramiento de nuestra sociedad con su trabajo productivo, con su esfuerzo, que puedan integrarse y ser personas de bien, en pocas palabras, es ayudarlos a que logren ser alguien en la vida" (EDF1).

En lo expresado en el fragmento anterior, encontramos una clave fundamental para comprender el sistema de ideas dominante alrededor del cual circulan los significados de la formación de ciudadanía en la escuela: "el ser alguien en la vida" le permitirá al joven convertirse en un adulto responsable, con carta de ciudadanía, a fin de integrarse socialmente, colocándose en la ruta marcada y teniendo como horizonte el respeto a la patria, a las leyes y a las instituciones. En este sentido, pareciera confundirse el proceso de ciudadanización con el logro de la movilidad social de los sujetos como aspiración.

Esa manera de entender la intencionalidad de la formación de ciudadanía resultó compartida por otros maestros y maestras que colocaban junto a la expresión de "ser alguien en la vida" otros rasgos, como: "ser responsable", "conocer las leyes", "ayudar a los demás" o "respetar nuestros símbolos patrios".

El análisis nos mostró que la expresión "ser alguien en la vida" se entretejía con otros sentidos que nos hablaban de una manera de pensar los modos mismos de pertenencia identificante, tal como se muestra en el siguiente fragmento en que una maestra comenta que aquel que ha logrado ser alguien en la vida, es quien: "ha ocupado un lugar dentro de la sociedad, a base de esfuerzo, a base de trabajo, a base de sacrificio" (EMF4).

Esa manera de significar la formación de ciudadanía revela el carácter restrictivo que la definición entraña, pues se construye en oposición a quien no ocupa un lugar y por

\footnotetext{
${ }^{4}$ En México, a través de la frase "ser alguien en la vida" se condensa el mito aspiracional a través del cual se vislumbra la esperanza de ser reconocido socialmente.
} 
lo tanto es excluido, razón por la cual se privilegia el polo de la integración frente al de la diferencia en la dinámica identitaria. Dicho de otro modo, los objetivos y estrategias de formación ciudadana que se propician en la escuela se orientan a reforzar procesos de identificación ciudadana de tipo atribuido (Dubar, 2002) que, como tales, favorecen una forma de integración estatutaria a grandes totalidades, aun cuando ello conlleve el sacrificio de la diferencia.

\section{Las estrategias de identificación ciudadana en la formación}

La reconstrucción de las estrategias permitió identificar que las tecnologías de gobierno que se despliegan en la institución están centradas en favorecer el desarrollo de determinadas formas de identificación ciudadana. Dichas estrategias apuntan a tres acciones a las que aludimos empleando los términos de los informantes: a) el "bombardeo de modelos"; b) "el choro sobre el futuro" ${ }^{5}$ y c) "la ocupación y el silenciamiento". Las estrategias funcionan poniendo orden ahí donde la diferencia produce confusión, impidiendo su manifestación y obstaculizando el trabajo del sujeto sobre sí mismo, con lo cual se evita conformar otro horizonte de ciudadanización. Ello no resulta extraño, sobre todo cuando lo que se busca es normalizar a través del ejercicio de la clasificación y de la aplicación del criterio de lo "absolutamente escolar". Empezaremos por este último.

Lo incoloro, inodoro e insípido: las actitudes escolares

A partir del análisis de las entrevistas realizadas a los maestros, encontramos múltiples referencias a la necesidad de prohibir prácticas relacionadas con las expresiones de afectividad entre los jóvenes, los usos de determinados peinados (como "la moda de los picos $\left.{ }^{\prime \prime}\right)$, vestimentas o adornos en el cuerpo (como el piercing). Frente a estas manifestaciones, los maestros plantean la necesidad de fortalecer criterios que distingan con claridad lo permitido de lo no permitido, y evitar esto último para lograr los propósitos formativos.

La expresión de la directora en el sentido de que los estudiantes debían mantener "actitudes absolutamente escolares", revela los márgenes que se construyen entre lo aceptable y lo no aceptable, y lo que se espera de los comportamientos de los jóvenes. Por su aparente asepsia, ese criterio funciona como marcador de identificación ciudadana y, a través de él, se construye una idea de normalidad que homogeneiza a los estudiantes al separarlos de su ser joven y de su derecho a manifestar diferencia. Al aplicar dicho criterio, se despoja al joven de cualquier signo que se considere

\footnotetext{
${ }^{5}$ Esta frase coloquial recuperada del discurso de los estudiantes se construyó como categoría etnográfica para analizar la valoración que los alumnos hacen sobre el discurso docente con respecto a la juventud y el futuro cuando se vuelve prescriptivo y moralizante, dejando de ser significativo en su formación.

${ }^{6}$ De este modo se refieren los maestros a los peinados que algunos jóvenes portan.
} 
contaminante de la labor formativa. Asimismo, posibilita el ejercicio de la acción educativa sobre el otro, con el fin de desplegar procesos de identificación de tipo atribuido que posibiliten la transmisión de los valores de nuestra idiosincrasia, dentro de un marco de homogeneidad democrática.

Sin embargo, si entendemos que lo que se marca a través del sistema exclusionario ${ }^{7}$ es no solo aquello que se descalifica y desconoce, sino también lo que se reconoce y se autoriza como libertad posible del sujeto, encontramos que el criterio de las "actitudes absolutamente escolares" engloba otros aspectos que resultan estratégicos para sostener la configuración de los márgenes de normalidad desde donde pueden ser pensados los procesos de identificación ciudadana. De allí, la relevancia que, para la interpretación, adquirió el siguiente fragmento:

"Lo que sentimos es que con todo lo que está pasando de promoción, publicidad o difusión de conocimientos de derecho hacia la gente, hacia los niños, hacia los papás, se ha olvidado un poco difundir ahora sí, que la contrapartida. [...] Nos dedicamos exclusivamente a promover los derechos de los niños, porque nos preocupó mucho que algunos papás abusaran de los niños, entonces, solamente vamos a promover los derechos de los niños. A un nivel de sociedad, en donde nada más se están dando a conocer los derechos, también se está cayendo en el abuso o en la exageración de "este es mi derecho y porque este es mi derecho, lo voy a pedir con gritos, y ahora te regreso la acusación". "iAh! Tú me estás diciendo algo porque traigo un fierro en la lengua, entonces yo te digo que es mi vida, que es mi cuerpo, que me respetes" entonces, le están gritando al papá, a la mamá, o al maestro, que por qué se mete con su cuerpo o con su vida; entonces, ¿qué es lo que va a entrar en el respeto hacia una sociedad? (EDF1).

A partir de este fragmento podemos encontrar un indicio importante en la manera de entender la formación de la ciudadanía en la escuela: desde la racionalidad dominante no se asumen los derechos humanos como pieza clave para la reconceptualización de los procesos de identificación ciudadana. ${ }^{8}$

Si relacionamos lo dicho en el fragmento anterior con la prohibición de ciertas conductas catalogadas como inaceptables entre los jóvenes (la moda de los picos, el piercing y manifestaciones de afecto y sexualidad entre ellos), encontramos que la idea de lo absolutamente escolar opera como criterio de clasificación que delimita el tipo de estrategias que propicia el dispositivo escolar para producir cierto tipo de

\footnotetext{
${ }^{7}$ Para Popkewitz (1998) es posible pensar que los discursos producen mapas que nos indican simbólicamente cómo ordenar los objetos del mundo para el escrutinio y la práctica a partir de sistemas de inclusión/exclusión específicos que nos aportan principios para razonar sobre las acciones y caminos a seguir en la procesos formativos en la escuela.

${ }^{8}$ Aquí vale traer a colación la siguiente afirmación de Cruz Pérez: "Lo cierto es que las personas cobramos nuestra identidad y estima en el seno de una comunidad que nos reconoce derechos o los niega, que nos hace saber que somos miembros suyos o nos hace sentirnos extraños. Si una comunidad política deja sin protección a alguno de sus miembros en sus derechos básicos, está demostrando con hechos que no lo considera en realidad como ciudadano suyo. Aquel a quien no se le trata como ciudadano tampoco se identifica a sí mismo como tal (2005: 3-4).
} 
subjetividades. Mediante su aplicación, se estrecha, casi hasta anularse, el margen para que los jóvenes puedan decidir sobre el propio cuerpo. Se trata, prácticamente, de la prohibición de tomar decisiones, que conlleva sanción en caso de no cumplirse. No aplicar el criterio significaría autorizar y legitimar al otro -el joven- como sujeto de derecho, con derecho a decidir sobre su cuerpo y, sobre todo, sobre su vida; con derecho a pedir respeto a sus propias decisiones.

Entendemos entonces el sentido que adquiere dicho criterio, como expresión del sistema exclusionario desde donde se plantean límites frente a la irrupción de las múltiples manifestaciones de lo juvenil. No aplicarlo significaría abrir las puertas hacia nuevas maneras de concebir al sujeto joven ya no como estudiante, sino como ciudadano en formación. Ello modificaría de manera drástica la relación saber-poder al interior del dispositivo, pues al integrar otras maneras de pensar la ciudadanización de los jóvenes, estas tendrían que articularse con acciones relacionadas con el reconocimiento, el cual se favorece cuando la posibilidad de decidir se incorpora en el repertorio de prácticas sancionadas como legítimas.

\section{El bombardeo de modelos}

Otra estrategia de identificación ciudadana reconocida en el análisis es la relacionada con el "bombardeo de modelos", según la frase dicha por una de las maestras entrevistadas:

"Yo creo que si mis alumnos me vieran llegar en un carro enorme, muy bien vestidita y demás, a lo mejor tendría la imagen de más respeto hacia mis alumnos o pensarían que daría más que pues, a lo mejor siendo responsable, siendo trabajador ¿No?. Entonces, como que esa es la dinámica en la que estamos inmersos. [...] Pero si hacemos ahora lo contrario, si los bombardeamos y les decimos o ponemos como modelos, ya no a los que sean muy, muy esbeltitos y bonitos, el problema se resolvería y tal vez ellos digan "No me detengo, a mí me preocupa y estoy investigando y estoy avanzando y estoy avanzando para ser alguien en la vida" (EMF 4).

Esta estrategia, si bien ha formado parte nodal de los procesos de identificación ciudadana que se propician en la escuela, tiende a actualizarse a fin de contrarrestar las influencias consideradas nocivas, que resultan de la difusión de determinados modelos de comportamiento por los medios de comunicación. Es un bombardeo que se contrarresta con otro bombardeo.

El bombardeo de modelos viene a ser una estrategia fundamental para favorecer la identificación. Con ella se busca contraponer los valores del trabajo y la responsabilidad como marcadores de identificación frente a aquellos otros que, ligados al éxito y la apariencia, resultan ser muy atractivos para los jóvenes $y$, por ello, a juicio de los profesores, se dificulta el logro de los objetivos pedagógicos previamente trazados, 
que como hemos visto anteriormente se articulan con la idea de "ser alguien en la vida a fin de ocupar un lugar".

Desde el razonamiento de la maestra la lucha ha de ser frontal debido a que lo que se pone en juego en la formación no son simples travesuras, sino, y sobre todo, la necesidad de preservar el respeto como valor fundamental que ha de garantizar la pervivencia de la estrategia del modelado en los procesos de identificación ciudadana que se realizan en la escuela.

Esta situación no resulta extraña, sobre todo cuando entendemos que tal manera de razonar sobre la formación, centrada en la necesidad de propiciar la identificación con determinados modelos a fin de impedir la reproducción/sobrevivencia de otros, tiene ya una larga historia dentro de nuestro pensamiento educativo.

Para Popkewitz (1998), la estrategia de 'reproducción del modelo de rol' se inscribe en el pensamiento pedagógico como concepto de regulación y de gobernación de las prácticas y, por lo tanto, como efecto de poder:

Históricamente, la idea de modelo de rol es un concepto de regulación y gobernación. Se trata de un concepto que surgió por primera vez en la teoría social de principios del siglo XX para explicar las relaciones de otros como "imitación-sugerencia". La imitación-sugerencia era "una forma de explicar la influencia de un líder o de un genio sobre el público, y las formas mediante las que se podía marginar a las mujeres y a los negros, al privarlos de 'copias' de la cultura dominante (Leys, 1994: 213 citado en Popkewitz, 1998: 67).

Desde la perspectiva de los autores, la relevancia del bombardeo de modelos como estrategia de formación radica en que, a través de ella, lo que se busca es privar a los jóvenes de cualquier otra copia que, lejos de la racionalidad escolar, pudiera introducir algún factor de diferencia.

En este contexto, la idea de bombardeo de modelos como estrategia formativa nos habla de la dificultad de la escuela para pensar al proceso de formación en medio del cambio más allá del horizonte de la continuidad cultural. En palabras de Puig (2005) podemos decir que el paradigma de la formación se actualiza al brindar el cartografiado de una ruta previamente trazada, a la vez que se elimina la posibilidad de introducir una brújula, la cual resultaría fundamental si se buscase que los jóvenes adquirieran las herramientas y habilidades para realizar el ejercicio de ponderación de la valía de los distintos modelos con que la sociedad bombardea, incluida la institución escolar. Es decir, si se quisiera contribuir a fortalecer la capacidad de decisión de los sujetos jóvenes.

La estrategia del bombardeo de modelos ayuna de esa capacidad de decisión, se presenta como una estrategia que promueve la identificación ciudadana de modo heterónomo y ello tiene dos efectos. Por una parte, refuerza los procesos de carácter 
heteroformativo (Yurén, 2005) y, con ello, debilita la vía autoformativa, y, por otro, obstaculiza la construcción de modos de pertenencia identificante de tipo reivindicado -que implicarían asumir que los jóvenes juegan un papel protagónico en el ejercicio de problematización y de ponderación sobre la pertinencia de los modelos que se les ofrecen actualmente. Lo que se reafirma son modos de pertenencia identificante de tipo atribuido. Estos últimos se presentan como finalidad y estrategia de la formación.

\section{El choro sobre el futuro}

Al analizar las entrevistas de los jóvenes encontramos múltiples referencias en torno al tipo de interacción que los maestros establecían con ellos, sin una intención verdaderamente comunicativa. Esta forma de interacción, calificada por algunos, de manera coloquial, como 'el choro', era cotidiana. A través del choro, los profesores intentaban, a su manera, infundirles el deseo de pensar en el futuro, pero también compartir su visión acerca de los problemas por los que atraviesa la sociedad actualmente, así como su percepción en torno de la situación de la juventud. Dicha estrategia, en la mayoría de los casos, era calificada por los jóvenes como poco eficaz, si no es que hasta contraproducente. Así lo describe una joven cuando recuerda a uno de sus maestros:

"Mi peor maestro fue uno de primero, porque nada más llegaba al salón y decía "De tal página a tal página. Pónganse a hacer", y no nos explicaba nada, y luego ya, si algún niño le preguntaba, ya nos echaba su choro y nos empezaba a decir que nosotros ¿a qué íbamos a esa escuela?, que había otros muchos niños que querían estar en ese lugar, que nosotros nada más íbamos a holgazanear [...] y que no, que nos iba a explicar eso pero rápido y que el que entendiera qué bueno, y quien no, a ver cómo resolvía sus cosas, y luego ya se ponía a platicar: "Es que ustedes los niños, no, los jóvenes, no ven lo que hacen. Por ejemplo, ya están contaminando nuestro país y que según ustedes son el futuro de México, yo no sé cómo pueden decir eso, si en Física no van bien, si en esto no van bien". Y así se le acababa su clase y ya nada más decía: "A ver, de esta página a esta página es de tarea", y ya, no nos explicaba, ni nos hablaba de su clase" (EJF1).

Según los jóvenes entrevistados, este tipo de interacción no les era útil y consumía, en algunas ocasiones y sobre todo con algunos maestros, gran parte del tiempo destinado a trabajar los contenidos de las materias. En un extremo, 'el choro' los desviaba totalmente del tema que originalmente se estaba tratando.

Comentarios de este tipo aparecieron en la mayoría de las entrevistas hechas a los estudiantes. Ello revela que existe una sobredimensionalización de la preocupación por los valores frente a lo académico, que se expresa a través de un discurso moral que desde lo que podríamos llamar 'la tradición de la conferencia' inunda al quehacer educativo. Dicho discurso tiene un tono prescriptivo y se moviliza al margen del objeto de enseñanza; además de que posee un tinte descalificador y enjuiciador de los 
comportamientos juveniles. Por eso, como plantea la joven, la estrategia resulta ineficaz.

En el siguiente fragmento podemos encontrar algunas claves que pueden ayudar a explicar el porqué del rechazo a esta estrategia:

"Los maestros hablan de nosotros, pero lo hacen en sus puntos de vista de ellos como profesores. Platican entre todos de los grupos, cómo los ven... pero no, no sé. Pero con nosotros no, solo de vez en cuando nos echan su choro, pero no nos sirve, porque pues no hacemos caso a nada de lo que nos dicen" (EJF 2).

Lo que resalta en el fragmento anterior es la descalificación del 'choro' como estrategia que pretende ser comunicativa, pues al colocarse el profesor en sus puntos de vista, acaba siendo monólogo que responde a una mirada parcial $\mathrm{y}$, además, sesgada por el afán prescriptivo. En este sentido, no es de extrañar que surja el reclamo por parte de los jóvenes, que conlleva la demanda de que los profesores se comuniquen con ellos, que se establezca un clima propicio al diálogo e intercambio de perspectivas que requiere del reconocimiento de los puntos de vista del otro.

Sin embargo, ello se vuelve difícil desde la racionalidad dominante que se deja traslucir a partir de lo analizado, pues lejos de interesar el desarrollo de la capacidad de los propios jóvenes para pensar acerca de lo que tendrían que hacer para construir su futuro, el énfasis se encuentra en la necesidad de que ellos se adscriban a la forma de ver el futuro que desde la idiosincrasia busca reproducirse. Por ello es que consideramos que lo que prevalece en el 'choro' sobre el futuro, es lo que Yurén y Araújo et ál. denominan la "cultura de la instrucción", que al estar basada en la prescripción, el adoctrinamiento y la inculcación, lo que se favorece es que:

El discurso se distribuya a manera de doctrina, es decir, como prescripciones por obedecer [...] los contenidos valorales sean tratados como declarativos o a lo sumo como contenidos actitudinales que deben internalizarse por socialización (2003: 637-638).

En este contexto podemos interpretar que a los maestros les resulta difícil hablar con los jóvenes, descentrándose de sus propios puntos de vista, debido a que ello requeriría dejar de verlos a través del tamiz que significa la aspiración de ser alguien en la vida, y atreverse a reconocer al otro más allá de la propia perspectiva. Si esto llegara a darse, se abriría un espacio para que el maestro pudiera reconocer la desazón de los jóvenes, su aburrimiento y su ejercicio crítico.

Ahora bien, desde la perspectiva de los jóvenes, la necesidad de entablar procesos más dialógicos con los maestros resulta relevante, debido a que les preocupa el futuro, tal como nos comentó un joven: 
"Ellos nada más se quedan en decirnos todo lo que debíamos de ser, pero que no somos, nada más en el futuro, nunca se preguntan qué queremos ser nosotros en nuestro futuro, qué necesitamos. Yo necesito saber de muchas cosas que me preocupan" (EJM7).

A partir de lo expresado, podemos inferir el interés de los jóvenes por hablar de su futuro, pero desde lo que son sus posibilidades en el presente. En este sentido, pareciera que lo que configura el desencuentro entre maestros y jóvenes es el destiempo que se abre entre los tiempos pedagógicos y los tiempos individuales en la formación.

En los relatos de los jóvenes, pareciera que la preocupación en torno a la necesidad de saber sobre el futuro, se relaciona precisamente con la dificultad para verse en el futuro. Así lo plantea uno de los jóvenes en el siguiente fragmento:

"Yo no me veo de grande más que alto, no tengo un plan a muy largo plazo, no me veo de grande. Supongo que, conforme voy viviendo debo de hacerlo, aunque tampoco soy de los que haga las cosas nada más por hacerlo, también lo pienso, digo, sería una tontería nada más actuar por actuar, no tendríamos cerebro pero, a largo plazo no, no me veo. O tal vez de adulto me vería como trabajando en una oficina, pero, teniendo muchos hobbies pues, yo no me veo casado, pues no sé, es una forma de amarrarme, no creo en el matrimonio, ni en los hïos" (EJM 9).

A partir de lo dicho por este joven, podemos interpretar que lo que se pone en juego es la necesidad de hablar de otra manera del futuro, más allá del horizonte prescriptivo que deriva de la idea de ser "alguien en la vida". Se trata del agotamiento de la idea misma de futuro, cuestión que no necesariamente significa que ellos no aspiren utilizando la expresión- a ser "alguien en la vida", sino que dicha expresión adquiere un sentido distinto al que le da la escuela relacionado con ocupar el lugar.

Haciendo una interpretación con respecto de la manera como se asumen en un futuro, lo que pareciera que marca la experiencia de los jóvenes es la ruptura de trayecto que aparece cuando ya no son capaces de reconocerse en el camino que el otro -sus padres, maestros y otros adultos- han podido construir para sí mismos. De allí que podamos comprender el sentido que adquieren las frases "yo no me veo a futuro, pero me preocupa saber del futuro" (EJM7), tal como lo comentó otro de los jóvenes entrevistados.

Se puede decir que lo que los jóvenes demandan es otro tipo de saberes y vías para construirse como sujetos que, lejos del 'choro', les permitan problematizar al mundo y a sí mismos dentro de ese mundo, que contribuyan a imaginarse nuevos horizontes de ciudadanización, que favorezcan una nueva ética de futuro en donde se propicie la posibilidad de hacerse cargo de sí mismos. 


\section{La estrategia de la ocupación y del silenciamiento}

En el análisis de las entrevistas realizadas a los jóvenes, pudimos documentar que, en la mayoría de los registros, ellos y ellas hacían una crítica a los métodos que utilizan los maestros centrados en la copia y el dictado. Para profundizar en el sentido que adquiere la crítica que las y los jóvenes realizan, traemos a colación el siguiente fragmento:

"Los temas que siento que más me preocupan ahorita y que me gustaría saber es todo lo que tiene que ver con la sexualidad. Pero nadie nos habla de eso, según [dicen] que en Cívica y Ética íbamos a hablar eso, de la sexualidad, pero lo único que nos está enseñando la maestra es del periodo pasado y ni enseña [...] Nada más haciéndonos que copiemos los métodos anticonceptivos, nada más" (EJF 2).

Desde la perspectiva de la joven, en la acción de copiar, si bien se obtiene alguna información, esta les resulta insuficiente, repetitiva y parcial, en la medida en que no les es útil para responder a sus preguntas; además, esa forma de trabajo cierra el espacio para el diálogo. En este contexto, pareciera que la acción de copiar resulta ser un placebo frente a las demandas de los jóvenes a sus maestros para que les hablen de eso: de sus preocupaciones. Se elimina con ello el riesgo de dar nombre a las experiencias que inquietan a los jóvenes ligadas a la sexualidad.

El reconocimiento de la insuficiencia e ineficacia de los métodos de la copia y el dictado para la formación, también fue compartida por la mayoría de los maestros y maestras entrevistadas, aunque a partir de otras razones, relacionadas con la necesidad de mantenerlos ocupados, tal como se puede ver en el siguiente fragmento:

"La idea de mantener ocupados a los sujetos ha surgido por el problema del grupo de tercer A, a ellos se necesita tenerlos todo el tiempo ocupados para controlar su inquietud. Esa es la conclusión a la que llegamos y lo que estamos haciendo. Pero no todos los maestros pueden hacerlo, porque son muy tradicionales, solo la copia y el dictado" (EMF4).

A partir de lo dicho por la docente en el fragmento anterior, podemos aclarar la intencionalidad que pareciera guiar la necesidad de introducir nuevos métodos de enseñanza por parte de los maestros: se trata de modificar los ritmos de enseñanza a fin de mantener ocupados a los jóvenes. En este sentido, la necesidad de superar los métodos tradicionales de copia y dictado -en contrasentido a las demandas planteadas por los jóvenes- se inscriben en la necesidad de construir estrategias de control sobre la inquietud de los jóvenes.

La idea de tener ocupados a los jóvenes se presentó de manera recurrente en las entrevistas realizadas a los maestros. Esto nos hizo ver la importancia que reviste esa acción, pues más allá de la intención de introducir nuevos métodos de enseñanza, 
supone la actualización de un tipo de estrategia de formación ciudadana que, centrada en la idea de ser alguien en la vida, se ve como posibilidad para "rescatar a los jóvenes" de un ambiente y de prácticas que se consideran nocivos. Una maestra lo expresa así:

"Yo los veo inmersos en otro mundo, en otro lado, pero no es el ámbito estudiantil, es otro, ellos tienen otros intereses menos la escuela. Para ellos la escuela no es, así, fundamental, por eso hay que rescatarlos de ese mundo" (EMF3).

Aquí vale decir que desde la perspectiva de los maestros existe un desinterés de los jóvenes con respecto a los aprendizajes que ofrece la escuela. Ese desinterés, dice uno de ellos "se ha venido manifestando de hace unos 7 u 8 años para acá", por eso, dice otra maestra, se ha vuelto "una lucha permanente y cada vez más fuerte, para que no se distraigan y capten atención, porque ellos ya no están interesados, y es muy estresante para nosotros" (EMF4). Como se desprende de estas afirmaciones, el mantener a los jóvenes ocupados se ve como una posibilidad de superar el desinterés que manifiestan.

Vemos, pues, que la introducción de nuevos recursos y métodos no significa transformar el proceso formativo, y mucho menos significa poner en cuestión los efectos de la copia. Tenerlos ocupados es actualizar la estrategia para lograr el cometido de que los jóvenes aspiren a ser alguien en la vida. Esto se puede apreciar en el siguiente fragmento:

"¿Qué significa esta estrategia [la ocupación]? Que los maestros son una parte imprescindible, y vuelvo a decir la palabra "imprescindibles" en las reacciones y en las respuestas de los niños. jAy, no! ¿Qué se ganan con que una Directora les pueda dar un sermón de veinte minutos o de una hora, si lo cotidiano es lo que los está favoreciendo o echando abajo a ese grupo? Hacerles solo ese escandalito no sirve solo" (EDF 1).

En el entendido de que los maestros siguen siendo una parte imprescindible en el proceso formativo, se ve necesario que los docentes cuenten con nuevas competencias para enfrentar la relación con los jóvenes en las condiciones actuales. Pareciera que al cambiar las prácticas para mantener ocupados a los jóvenes, se tiene un afán modernizante que lucha contra la tradición pero, paradójicamente, la práctica se reinventa para no modificar los objetivos pedagógicos. Estos quedan incólumes. Dicho de otra manera, para estimular que los jóvenes aspiren a ser alguien en la vida, resulta más eficaz la estrategia de la ocupación en el quehacer cotidiano del trabajo grupal, que lo que se podría lograr con un "sermón de 20 minutos o un escandalito".

En la crítica que se hace al 'choro' cuando se ve como estrategia formativa, encontramos que lo que se está criticando es el modo de pensar la formación con un sesgo altamente moralizante, pero que propicia la heteronomía. La crítica se funda entonces en que, al privilegiar el choro, se deja de lado la búsqueda de estrategias que 
favorezcan con eficacia la construcción de lo que Popkewitz (1998) llama la "autogobernación del sujeto".

Pero ¿qué virtudes esconde la estrategia de la ocupación para considerarse tan eficaz a fin de actualizar los propósitos formativos escolares ligados a la idea de ser alguien en la vida? En el discurso de los maestros podemos encontrar indicios acerca de por qué la estrategia resulta tan relevante. Se trata -dice una maestra- "de que su cabecita no esté en otro lado y eso se da porque nada más están así solos, desairados, solitos y pensando en cosas, pues malas [...] sino que siempre estén ocupados haciendo alguna actividad positiva" (EMM 2).

En esta lógica y a fin de impedir que los jóvenes piensen cosas malas es necesario combatir "la ociosidad que es la madre de todos los vicios". Esta fue la expresión empleada por una de las prefectas, después de corretear jóvenes que se habían salido del salón y buscaban dónde esconderse, y de haber logrado ordenar a los grupos en los que por alguna razón había faltado el maestro.

Tal como plantea Foucault, la utilización exhaustiva del tiempo se sustenta no solo en el principio negativo de no ociosidad, sino como economía positiva:

[... se] plantea el principio de una utilización teóricamente creciente siempre del tiempo: agotamiento más que empleo; se trata de extraer, del tiempo, cada vez más instantes disponibles $y$, de cada instante, cada vez más fuerzas útiles. Lo cual significa que hay que tratar de intensificar el uso del menor instante, como si el tiempo, en su mismo fraccionamiento, fuera inagotable; o como si, al menos, por una disposición interna cada vez más detallada, pudiera tenderse hacia un punto ideal en el que el máximo de rapidez va a unirse con el máximo de eficacia (1996: 158).

En este sentido, la ocupación como estrategia para favorecer la identificación ciudadana favorece la acción de fabricar ciudadanos desconectados de sus propios intereses. Al ponerla en acción, se trata de impedir que los jóvenes piensen cosas malas. Se busca, así, el control del pensamiento.

Desde esta perspectiva, la ocupación de los jóvenes en diversas tareas tiende a verse como una estrategia integral que, además de introducir otro ritmo didáctico a la clase, permite al maestro controlar el movimiento. Así lo comentó una joven entrevistada:

"Yo creo que el principal problema que vivimos en la escuela es que con cualquier cosita, como en las clases, con cualquier cosita ya nos distrajimos, porque como que nos... algo dentro de nosotros nos dice "Muévete o voltea, o ríete" o algo así, entonces, algo va, algo que va más con nuestro [modo de ser, en lugar] de quedarnos quietos, o de poner atención. Entonces por eso. Ese es el problema que yo siento que ahorita estamos enfrentando, [...] pero yo siento, solo no se mueven los muertos. Nosotros estamos vivos, tenemos que seguir, que vivir la vida, entonces, aunque nos quedemos quietos, quietos, quietos, a fuerzas en un momento te tienes que mover o te 
tienes que reír o tienes que hacer algo para que te sientas a lo mejor bien con tu organismo, contigo mismo, que ya sentiste que ya moviste un pie, que ya moviste esto, ya te reíste, ya volteaste a ver a tu compañero, o cosas así" (EJF1).

Resalta en el testimonio de la joven la oposición entre la demanda de los maestros para que estén quietos y lo que los jóvenes quieren. Para ellos, la quietud no es otra cosa que la muerte, por lo que sus comportamientos los asocian con el movimiento, que es la vida. En este contexto, mantener ocupados a los jóvenes significa detenerlos, silenciarlos, pero también apretarlos, como se puede apreciar en el siguiente fragmento:

"Yo me veo como adolescente con muchos problemas con todos. Porque todo el mundo te empieza a apretar. En mi familia y en la escuela me empiezan a decir que por qué 'esto' y 'esto' y antes era 'esto'; te comparan mucho como ahorita a cuando tenía doce años. [...] Se me castiga... bueno, mi mamá me ha castigado muchas veces porque no me da permiso de irme a jugar y yo siento que es importante ir a jugar... me dijo que... me castigó de esa manera y yo le dije: "me podrás encerrar, pero, pues, yo seguiré pensando en eso". De ahí han surgido problemas conmigo, por eso" (EJF 4).

Entendemos entonces el sentido que adquiere la ocupación como estrategia. Se trata, utilizando el lenguaje coloquial, de no dejarlos ni respirar. Se pretende aquietar el pensamiento a fin de que los jóvenes no piensen lo impensable, pero también de la quietud del cuerpo para impedir cualquier manifestación de movimiento y con ello de diferencia, a fin de favorecer el proceso de desconexión de la pulsión de alteridad. En este sentido, la ocupación opera como acción de matar el deseo, lo cual no significa otra cosa que la renuncia de sí mismo, tal como plantea Foucault (1996).

Pese a todo, siempre existe un resquicio que puede ser aprovechado, como reconoce la joven cuando afirma: "me podrás encerrar, pero, pues, yo seguiré pensando en eso".

\section{Conclusiones}

La investigación realizada nos permitió avanzar en el conocimiento y análisis del modo como se configuran las prácticas de la formación de ciudadanía en la escuela secundaria y la forma como estas se producen, reproducen y organizan, a fin de propiciar determinados procesos de identificación ciudadana. Tal como ha sido expuesto, la constelación de significados que dan sentido a las prácticas de la formación de ciudadanía en la escuela se entretejen alrededor de la ideología aspiracional de "ser alguien en la vida" a fin de ocupar un lugar.

Dicha intencionalidad desplaza la posibilidad de interacciones fundadas tanto en la asunción de que el otro -el joven- es sujeto de derecho, como en el reconocimiento de la diferencia, y actúa como mecanismo fundamental de control simbólico a partir del 
cual se diferencia lo pensable de lo impensable en el campo de la formación de ciudadanía en la escuela, orientándola exclusivamente hacia la conformación de procesos de identificación ciudadana de tipo atribuido que se organizan a través del principio de lo "absolutamente escolar".

La importancia que reviste la asepsia de dicho principio como marcador de identificación ciudadana, es que a través de él se construye una idea de normalidad que homogeneíza a los estudiantes al separarlos de su ser joven. La finalidad de dicho principio es obturar la manifestación de cualquier diferencia, debido a que ello supondría legitimar el derecho a decidir de los jóvenes en tanto "sujetos de derecho".

Es a través de este principio, en conjunción con diversas estrategias de formación, que se dinamiza la construcción de determinados procesos de identificación ciudadana, a saber: a) el bombardeo de modelos, b) el choro sobre el futuro y la juventud y c) la ocupación y el silenciamiento. Lo que dichas estrategias comparten es que se dirigen a obturar la confusión que produce la demanda de reconocimiento que manifiestan los jóvenes, delimitando el modo a través del cual el sujeto puede pensarse a sí mismo en desconexión de sus vivencias, necesidades y preguntas.

De manera específica, a través del bombardeo de modelos, lo que se intenta es reproducir el rol a través de la copia, buscando que los alumnos sigan la ruta previamente trazada, el ejemplo es la herramienta.

Con respecto al 'choro' sobre el futuro y la juventud, en dicha estrategia el uso del discurso prescriptivo es la fuente a partir de la cual se pretende que los alumnos se convenzan de la valía de seguir el camino indicado. En relación con la ocupación, ella requiere de la actividad constante a fin de silenciar la palabra y detener aquel movimiento continuo, libremente experimentado, para dirigirlo hacia otro lugar que lo haga susceptible de ser controlado.

Es así que los efectos que dichas estrategias producen van en detrimento del ejercicio de elección de los jóvenes y de su capacidad de problematizar la realidad presente para construir una idea propia de futuro, e impiden que los jóvenes puedan producirse a sí mismos como sujetos ciudadanos asumiendo que pueden intervenir en la modificación del orden social.

\section{Referencias bibliográficas}

Cruz, P. (2005). La educación ética para el ejercicio de la ciudadanía, En: Arredondo, A. y Góngora, J. (Coords.) Educación, ética y responsabilidad ciudadana de los docentes. México: IFE, UPN, UAEM.

Dubar, C. (2002). La crisis de identidades. La interpretación de una mutación. España: Ediciones Bellaterra.

Foucault, M. (1988). Hermenéutica del sujeto. Madrid: La Piqueta.

Foucault, M. (1996). Vigilar y castigar. Nacimiento de la prisión. México: Siglo XXI.

Foucault, M. (1998). La verdad y las formas jurídicas. México: Gedisa. 
Foucault, M. (2003). Historia de la sexualidad, vol. 2: el uso de los placeres. México: Siglo XXI.

Goetz, J.P. y Lecompte M.D. (1988). Etnografía y diseño cualitativo en investigación educativa. Madrid: Morata.

Popkewitz, T. (1998). La conquista del alma infantil. Política de escolarización y construcción del nuevo docente. Barcelona: Ediciones Pomares.

Puig, J. M. (2005). Prácticas morales. Una aproximación a la educación moral. Barcelona: Editorial Paidós.

Yurén, T. y Araujo, S. (2003). La asignatura "Formación cívica y ética" en la secundaria general, técnica y telesecundaria. Su sentido y condiciones de desarrollo en el Estado de Morelos. México, Secretaría de Educación Pública, 2005. Colección Programa de Fomento a la Investigación Educativa. Informes finales de investigación educativa (Convocatoria 2002). Vol. IX, 219 pp. Disponible en: http://basica.sep.gob.mx/dgdgie/cva/sitio/pdf/materiales/resenas2002/pdfs/9Ter esaYuren.pdf fecha de acceso: 3 de agosto de 2005

Yurén, T. (2005). Ethos y autoformación en los dispositivos de formación de docentes. En Yurén, M.T., Navia, C. y Saenger, C. (Coords.) Ethos y autoformación del docente, Barcelona: Ediciones Pomares. 